Études Études de lettres

de Lettres

2| 2017

Du Rhin à l'Oronte: Maurice Barrès écrivain

\title{
De Venise à Venise
}

Étienne Barilier

\section{OpenEdition}

Journals

Édition électronique

URL : http://journals.openedition.org/edl/998

DOI : $10.4000 /$ edl. 998

ISSN : 2296-5084

\section{Éditeur}

Université de Lausanne

Édition imprimée

Date de publication : 15 septembre 2017

Pagination : 15-34

ISBN : 978-2-940331-65-9

ISSN : 0014-2026

\section{Référence électronique}

Étienne Barilier, « De Venise à Venise », Études de lettres [En ligne], 2 | 2017, mis en ligne le 15 septembre 2019, consulté le 16 décembre 2020. URL : http://journals.openedition.org/edl/998 ; DOI : https://doi.org/10.4000/edl.998

(c) Études de lettres 


\section{DE VENISE À VENISE}

Maurice Barrès a écrit La Mort de Venise; et Thomas Mann, quelque dix ans plus tard, La Mort à Venise. Le premier a vu ou a voulu voir dans la ville mythique le lieu de la déliquescence et de la mort, en face duquel son moi se découvre d'autant plus vivant et jouit d'autant plus de lui-même. Venise, donc, le conforte. Mais Venise, tout aussi mortifère, déchire et dévaste Aschenbach, le héros de Mann. Cette ville n'est pas pour lui l'occasion d'un triomphe, mais d'un échec et d'une catastrophe. Aschenbach prend conscience que la mort et les abîmes ne sont pas hors de lui, mais en lui. Barrès se mire dans Venise. Le héros de Mann va s'y détruire. Ce sont là deux formes de conscience, deux manières d'affronter les "puissances souterrraines».

Comparer Barrès à Thomas Mann, en dépit de l'extrême ressemblance des titres de deux œuvres dont ils sont les auteurs (La Mort de Venise et La Mort à Venise), peut paraître aventureux. Si je m’y risque néanmoins, c'est que bien avant moi, un très grand lecteur et critique français s'est permis de le faire, et l'a fait d'une manière saisissante. Il s'agit de Charles Du Bos, dans un discours de remerciement qu'il adressa à Thomas Mann en personne, lors de la visite de ce dernier à Paris, en 1926. Visite symbolique et chargée de sens, sept ans seulement après la fin de la Première Guerre mondiale. Un autre critique français d'alors, Edmond Jaloux, avait repéré dans l'œuvre de Thomas Mann ce qu'il avait nommé "un attrait germanique pour la mort». Or Du Bos affirme que cet attrait n'est pas germanique seulement. Car Maurice Barrès, lui aussi, possédait "un sens aigu des puissances souterraines" ${ }^{1}$. Et la ressemblance entre les deux titres, La Mort de Venise et La Mort à Venise, qui n'est peut-être pas entièrement fortuite, est pour Du Bos l'occasion de ce rapprochement.

I. Cité par Ch. Du Bos, «Hommage à Thomas Mann», p. 671. 
Le plus remarquable est que l'auteur des Approximations ne s'est pas contenté de comparer deux rêves vénitiens, hantés par la mort, ni même deux esthétiques. Il s'est demandé si l'évolution de Maurice Barrès pouvait se comparer avec celle de Thomas Mann, lequel, après la guerre, était passé d'un nationalisme fort affirmé à un irénisme démocratique non moins affirmé. Certes, sa Mort à Venise paraît bien éloignée de ces questions, qui relèvent de la politique, fût-ce au sens le plus large et le plus haut du terme. Pourtant Du Bos le savait bien, et nous le savons avec lui: l'esthétique est toujours beaucoup plus que l'esthétique. Toute œuvre emporte avec elle une vision du monde, et l'obsession de la mort vénitienne, beau thème littéraire assurément, révèle certaine manière d'appréhender la vie humaine, la vie tout entière. La question sous-jacente que posait Du Bos était alors celle-ci: l'attrait pour la mort, qui caractérisait autant le Barrès de La Mort de Venise que le Thomas Mann de La Mort à Venise pouvait-il signifier non seulement une fascination pour la mort subie, mais aussi pour la mort donnée? Et comment échapper à cette fascination-là?

Thomas Mann semblait y avoir échappé. Après avoir chanté de manière carrément alarmante, durant la guerre, la «noblesse de l'irrationnel ${ }^{2}$ », il plaçait désormais son idéal dans une pensée éclairée par la raison, et dans ce qu'il appelait, d'un mot très fort sous sa plume, la "sympathie» entre les peuples, et leur réciproque "admiration». Il avait donc combattu victorieusement, en lui, certaines tentations mortifères; il était remonté, de la nuit tardo-romantique, jusque dans la lumière goethéenne. On sait qu'il en témoignera avec énergie et courage durant l'entre-deux-guerres, puis au cours de la Deuxième Guerre mondiale, par ses textes antinazis, ses éloges vibrants de la démocratie, et son Docteur Faustus, qui montre, somptueusement et sinistrement, où peut conduire une trop grande complicité de l'homme avec ces «puissances souterraines» dont parlait Edmond Jaloux. Adrian Leverkühn, le héros du Docteur Faustus, pactise avec le diable, à seule fin de créer des œuvres d'art, mais il se fait, d'une manière indirecte, réelle tout de même, le complice d'une politique et d'une action diaboliques. Thomas Mann était un fervent admirateur de Goethe; selon Du Bos, l'amour

2. Th. Mann, Gedanken im Kriege, cité in R. Cheval, Romain Rolland, L'Allemagne et la guerre, p. 359. La "civilisation", au contraire de la "culture" allemande, est «raison, démystification, ordre moral, décomposition, esprit». 
de la lumière, l'apollinisme et l'universalisme de l'auteur de Faust ont contribué à arracher aux démons de la nuit celui qui deviendra l'auteur du Docteur Faustus. Or, nous assure du Bos, Maurice Barrès était lui aussi un admirateur fervent de Goethe. Et il était en passe, lui aussi, de quitter un trop équivoque attrait pour la mort, afin de rejoindre le lumineux irénisme goethéen. Il était «en marche» vers un «idéal de sympathie ${ }^{3}$ qui l'aurait arraché définitivement à la fascination de la nuit, de l'abîme et du chaos. Un ordre lumineux, un lucidus ordo (formule que Du Bos emprunte à l'Art Poétique d'Horace) ${ }^{4}$ dont il ne lui suffisait plus qu'il «présidât à son art seul. Il voulait davantage, et sur le plan humain et sur le plan religieux, et, en eût-il eu le temps, je suis persuadé qu'il en serait venu à vouloir davantage aussi sur le plan des relations des peuples entre eux; il est mort dans l'état de la plus pathétique aspiration ${ }^{5}$.

Mon intention, dans cet exposé, est d'éprouver, si je puis dire, cette généreuse espérance de Du Bos, à la lumière, violente et sombre, de ces deux œuvres si proches et si éloignées l'une de l'autre, La Mort de Venise de Barrès et La Mort à Venise de Thomas Mann. Bien entendu, moins encore que Du Bos (car j'y suis infiniment moins autorisé que lui, faut-il le dire) je ne me permettrai de tirer des conclusions définitives, et de trancher sur ce que Barrès serait devenu s'il avait vécu. Mais il se peut qu'une écoute attentive de son œuvre vénitienne, suivie d'une écoute de celle de Thomas Mann, nous aide à savoir si oui ou non Barrès aurait effectivement pu se rapprocher, un jour, du lucidus ordo; s'il aurait pu écrire cette phrase que Thomas Mann écrivit à propos de Knut Hamsun tenté par le nazisme: "Il compromet sans remède son propre génie de poète par son attitude politique, ou plutôt par son manque d'humanité» ${ }^{6}$.

Cependant, je ne prétends pas ici comparer les professions de foi de Thomas Mann avec les professions de foi de Maurice Barrès. Ce sont bien deux ouvres d'art qui retiennent mon attention, La Mort de Venise, et La Mort à Venise. C'est en elles que je voudrais saisir sur le vif une vision du monde, une manière d'être au monde, une relation à la mort,

3. Cf. Ch. Du Bos, «Hommage à Thomas Mann», p. 674.

4. Cf. Horace, Ars poetica, v. 41.

5. Cf. Ch. Du Bos, «Hommage à Thomas Mann», p. 675.

6. Cf. Th. Mann, La victoire finale de la démocratie, p. 12. Plus loin, il écrit que «la démocratie [...] plus que tout autre régime, est déterminé[e] par le sentiment et la conscience de la dignité humaine» (p. 18 sq.). 
qui pourra peut-être me permettre de peser au trébuchet cette phrase et cette espérance de Du Bos: "Je suis persuadé qu'il en serait venu à vouloir davantage aussi sur le plan des relations des peuples entre eux". Avec cette idée que les écrivains sont indivisibles, et tout entiers dans tout ce qu'ils écrivent, que ce soient des romans, des essais - ou des professions de foi.

$$
* * *
$$

On objectera peut-être qu'il faudrait malgré tout comparer ce qui est comparable. La Mort de Venise, de Barrès, est écrit à la première personne, et relève peu ou prou de l'essai méditatif, tandis que La Mort à Venise, de Thomas Mann, est un roman. Je pourrais répondre que le livre de Thomas Mann est la transposition romanesque, passablement fidèle, et même plus fidèle qu'on ne peut dire, d'une expérience personnelle de l'auteur, mort non comprise. Et que de son côté, Barrès fait de Venise le lieu d'une méditation aussi libre que peut l'être un roman, et que le sont ses romans. D'ailleurs, un critique barrésien n'affirme-t-il pas que "le récit de voyage sera le modèle quasi exclusif des romans de Barrès " ${ }^{7}$ ? Mais encore une fois, la vraie réponse à cette objection de méthode est que tout auteur est tout entier dans chacune de ses œuvres, au-delà des genres adoptés; tout entier, en toute liberté créatrice.

Avant de me lancer dans ma tentative, je me suis évidemment demandé si la littérature critique s'était penchée, avec Du Bos, et après Du Bos, sur une comparaison entre la Venise de Thomas Mann et celle de Barrès. Sans être sûr, le moins du monde, de n'avoir rien manqué, je dois dire que je n'ai trouvé, sur ce thème précis, qu'un chapitre d'un ouvrage en allemand, déjà ancien, de Hellmuth Petriconi, qui s'intitule Das Reich des Untergangs ${ }^{8}$ ("Le royaume du déclin»); un chapitre intitulé précisément: "La Mort de Venise und Der Tod in Venedig». Mais hélas pour moi, ce chapitre ne fait guère que juxtaposer les deux œuvres sans jamais les comparer, et son but essentiel, d'ailleurs assez piquant, est de démontrer que le thème de la Venise décadente et pourrie est purement littéraire, purement fantasmé, et ne correspond à aucune réalité. Ce qui, nous allons le voir, n'est pas tout à fait faux... Mais à coup sûr, je me trouvais réduit à mes propres forces, et qu'on pardonne au barrésien

7. Cf. E. Godo, La légende de Venise, p. 18.

8. Cf. H. Petriconi, Das Reich des Untergangs, p. 67-95. 
très amateur que je suis si des textes importants m’ont échappé sur ce sujet.

\begin{abstract}
$* * *$
C'est à Venise que j'ai décidé toute ma vie [...]. Sur cette rive lumineuse, je crois m'être fait une idée assez exacte de ces délires lucides que les anciens éprouvaient au bord de certains étangs 9 .
\end{abstract}

Ce texte de Barrès date de 1888 , après trois voyages qu'il fit dans la cité des Doges entre 1887 et 1888. C'est alors qu'il lut Théophile Gautier, Taine et Stendhal, et qu'il découvrit les grands maîtres de la peinture vénitienne, Véronèse, Titien, Giambattista Tiepolo. C’est là qu'il écrivit une partie d'Un homme libre, ouvre dans laquelle on peut lire ces phrases: "J'étais prêt à m'aimer, à me comprendre jusque dans mes ténèbres: pour me guider, je comptais sur Venise» ${ }^{10}$. Ou bien: «Et cette cité abstraite, bâtie pour mon usage personnel, se déroulait devant mes yeux clos, hors du temps et de l'espace..." ${ }^{11}$. Ou encore: "Mon être et l'être vénitien sont illimités». Barrès était très jeune encore: 27 ans; et cela se sent dans ces phrases d'un égotisme aussi démesuré que naïf.

Lorsqu'il publia La Mort de Venise, sous-titre et chapitre principal du recueil intitulé Amori et dolori sacrum, du temps avait passé. Nous étions en 1903. Il avait 41 ans. Ce qui distingue cependant cet ouvrage des pages vénitiennes antérieures, outre un narcissisme un peu moins ingénu, c'est qu'elles font de la mort et de Venise de parfaits et constants synonymes.

Assurément, ce n'est pas la première fois, tant s'en faut, que Barrès médite sur la mort. Mais c'est peut-être la première fois qu'il chante sur cent pages un long thrène funèbre, dont il nous assure que la mélodie lui est offerte et imposée par Venise même. Et pour mieux nous en convaincre, il n'aura de cesse d'invoquer le témoignage de grands prédécesseurs: je ne suis pas seul à penser ainsi, à sentir ainsi, à identifier Venise à la mort, plaide-t-il: toute la haute littérature, toute la haute musique, toute la haute peinture m'ont précédé, et je ne fais qu'orchestrer tant de déplorations antérieures. Les maîtres et seigneurs de Venise, ce

9. Article publié dans La Batte, en octobre 1888; texte retrouvé par Henri Massis et publié dans les Nouvelles Littéraires du 18 août 1962.

Io. Cf. M. Barrès, Un homme libre, p. 154.

II. Ibid., p. 158. 
sont ceux-là mêmes qui sentirent et proclamèrent sa mort: "Wagner, Taine, Gautier, Léopold Robert, Sand, Musset, Byron, Chateaubriand et Goethe forment son "Conseil des Dix" ", écrit-il. Il ajoute d'ailleurs, pour le cas où nous aurions un doute: " - Ils ne sont que neuf, me dit un lecteur. - Qu'on réserve le dixième siège. Je connais telle candidature» ${ }^{12}$.

En réalité, ces artistes n'ont pas tous chanté la mort de Venise, expressis verbis. Il s'en faut de beaucoup. Leur relation à cette mort est souvent bien indirecte, voire inexistante. Ainsi, si George Sand fait partie du "Conseil des Dix", ce n'est pas parce qu'elle aurait chanté la mort de Venise, mais parce qu'elle vint dans cette ville avec Musset, et parce que "le génie de ce poète ne se témoigna jamais mieux que lorsqu'il subissait des reprises de la malaria vénitienne ${ }^{13}$. De même, et de manière plus étonnante encore, Barrès voit dans l'œuvre lumineuse de Tiepolo "la tristesse physiologique, l'épuisement de Venise» ${ }^{14}$. Quant à Léopold Robert, ce n'est pas son œuvre mais sa vie qui parle de mort à Barrès, puisque ce Chaux-de-fonnier s'est suicidé au palazzo Pisani, à cause d'un amour malheureux pour Charlotte Bonaparte, et parce qu'il écrivit: "Je me sens malade du mal de ceux qui désirent trop " ${ }^{15}$.

Le cas de Wagner est plus subtil, mais non moins significatif d'une tendance barrésienne à enrôler sous la bannière de la mort tous les grands visiteurs de Venise. Wagner, en réalité, n’a jamais décrit Venise comme un lieu de mort. Il est vrai qu'il y est lui-même décédé, mais enfin, ce n'est pas la même chose. Il est encore vrai qu'il écrivit à Venise une partie de Tristan et Isolde, œuvre dont on ne peut nier qu'elle ait quelque complicité avec les "puissances souterraines». Mais tout de même, Tristan ne se veut pas un portrait de Venise et n'a pas été suscité par Venise.

Il est alors frappant de voir de quelle subtile manière Barrès annexe Wagner. Mais pour bien le comprendre, il nous faut remonter jusqu'à Goethe, et à son voyage en Italie, en 1786. À Venise, Goethe entendit des gondoliers échanger, en chantant, des strophes du Tasse. Eh oui, rien de moins ! Il dit son admiration pour ce chant, et parle à son sujet de

I2. Cf. M. Barrès, Amori et dolori sacrum, p. 107.

I3. Ibid., p. 75.

I4. Ibid., p. 15.

I5. Ibid., p. 79. 
"plainte sans tristesse» ${ }^{16}$. Il ajoute que Jean-Jacques Rousseau, en son temps, avait noté ces mélodies. Soixante-douze ans plus tard, Wagner, séjournant à Venise, écrivit à Mathilde Wesendonck que «les stances du Tasse n'accompagnent plus le chant comme jadis» ${ }^{17}$. En effet, en 1858, les gondoliers chantaient toujours, mais sans paroles. Néanmoins, pour Wagner, ce manque n'en est pas un. Il signifie, par métaphore, ou par anticipation, que les paroles sont absorbées par la musique. Ce phénomène le ravit et le fascine, puisque cette absorption consonne, si l'on ose dire, avec son propre idéal de compositeur. Mais voici ce que le double texte de Wagner et de Goethe devient sous la plume de Barrès:

Sur le vaste miroir que la lune pâlissait, Jean-Jacques, puis Goethe, entendirent de l'une à l'autre rive deux chanteurs alternés se jeter les vers du Tasse ou bien de l'Arioste. Plainte sans tristesse [ici, Barrès cite donc Goethe - sans le dire]. Ces voix lointaines ont quelque chose d'indéfinissable qui émeut jusqu'aux larmes. Une personne solitaire chante pour qu'une autre animée des mêmes sentiments l'entende et lui réponde. Le Tasse et l'Arioste se taisent aujourd'hui ${ }^{18}$.

Or, dans le contexte de La Mort de Venise, il faut entendre: cette disparition des paroles est un signe de la disparition progressive et fatale de Venise même. Il n'y avait rien de tel, bien sûr, ni chez Goethe, ni chez Wagner, encore moins chez Rousseau.

Donc, à chaque fois, les grands exemples que Barrès invoque sont sinon dénaturés, du moins réinterprétés dans le sens d'un pressentiment de la mort de Venise qui n'existait pas chez les auteurs invoqués. Le seul cas, peut-être, qu'on pourrait citer, où Barrès reprend des formules qui disent en effet la mort de Venise, et précisément en lien avec la disparition des poèmes du Tasse du chant des gondoliers, voire de ce chant lui-même, c'est celui de Byron. Voici ce qu'écrit le poète anglais dans le chant IV de son Pèlerinage de Childe Harold, publié dans la deuxième décennie du XIXe siècle:

I6. "Eine Klage ohne Trauer». Cf. J. W. Goethe, Voyage en Italie, I, p. 172.

I7. Cf. R. Wagner, Lettre à Mathilde Wesendonck, du 5 sept. 1858.

I8. Cf. M. Barrès, Amori et dolori sacrum, p. 16 sq. 
À Venise, les chants du Tasse n'ont plus d'échos, et le gondolier rame silencieux; ses palais tombent en ruine sur le rivage, et il est rare que la musique s'y fasse entendre; à Venise, ces temps ne sont plus ${ }^{19}$.

Plus loin, cependant, une autre strophe de Childe Harold nous montre que la vision de Byron est loin d'être aussi mortifère que celle de Barrès. Voici qu'il évoque, un siècle avant notre auteur, la Venise des écrivains et des artistes; mais leur magie lui permet précisément de surmonter l'impression de fin de règne qui l'a d'abord saisi (qui est surtout la fin du règne terrestre et matériel de la République de Venise) :

L'art magique d'Otway, de Radcliffe, de Schiller, de Shakespeare, avait gravé dans mon esprit son image. [...] Je puis la repeupler à l'aide du passé [...]; et parmi les jours les plus heureux qui sont entrés dans la trame de mon existence, il en est, ô Venise ! qui se sont teints de tes couleurs $^{20}$.

Même Byron dément donc cette idée que Venise n'est que mort. D'ailleurs Ruskin déjà, dans Les Pierres de Venise, tordait le cou à la légende: "La Venise de la fiction et du drame moderne», écrivait-il, "est une chose née d'hier, une floraison de décadence, un rêve théâtral que le premier rayon du jour réduit en poussière ${ }^{21}$.

Le jeune Proust, dans sa préface à sa traduction de la Bible d'Amiens, du même Ruskin, reproche à Barrès de n'avoir pas inclus cet auteur dans son Conseil des Dix ${ }^{22}$. Mais on comprend assez pourquoi : Malheur aux démystificateurs!

Bref, Barrès a besoin que Venise lui parle de mort. Tout est bon qui nourrit ce discours funèbre. Il nous entretient à satiété, pour ne pas dire ad nauseam, d' épuisement", de "moisissure", de "corruption", d' "empoisonnement», de "malaria», de "millions de bactéries", de "cloaques", de "décompositions", de "fièvres", de "liquéfactions

19. Cf. G. G. Byron, Le Pèlerinage de Childe Harold, IV, 3, p. 395.

20. Ibid., IV, 18-19, p. 399.

2I. J. Ruskin, Les Pierres de Venise, p. 39.

22. J. Ruskin, La Bible d'Amiens, p. 81. 
de la mort». L'auteur s'avoue fasciné par ce qu'il appelle "la vie d'un cadavre" ${ }^{23}$.

Et pourquoi cette fascination? La réponse est assez simple: cette "agonie prolongée» le "séduit» ${ }^{24}$. Et «tant de beautés qui s'en vont à la mort nous excitent à jouir de la vie» ${ }^{25}$. On aurait donc tort de croire que ce chant à Venise pourrissante est la continuation du poème des Fleurs du mal, «Une Charogne». Car chez Baudelaire, la mort est l'irréductible. De la beauté, de la jeunesse et de la vie, elle est la limite horrible, la négation sans recours. Le spectacle de la charogne est le moins récusable des memento mori. Baudelaire dit l'horreur d'une vérité insupportable, dont la seule issue est une foi platonicienne dans la forme qui survit à son incarnation charnelle. Chez Barrès, la mort n'est que le repoussoir de la vie. Dès lors, la pire pourriture n'est pas un memento mori, mais un memento frui: souviens-toi de jouir. Plus que le souvenir de Baudelaire, il faudrait sans doute évoquer la proximité d'un Huysmans, d'un Jean Lorrain, voire d'un Mirbeau, d'un Loti, d'un Farrère, et du décadentisme fin de siècle. Mais je ne puis m'y attarder ici.

Une chose est sûre: depuis Un homme libre, où Barrès disait que Venise n'existait que pour son usage personnel, rien n'a vraiment changé: «Efface-toi, Venise ondoyante et bariolée. [...] tu nous deviens inutile; tu n'es que conséquence et nous sommes l'essentiel, le principe» ${ }^{26}$. Ce qui compte, c'est que le moi puisse retourner à lui-même et continuer à se rendre "culte». À se prolonger, à se projeter, à s’aimer dans Venise, à se contempler dans son miroir vert-de-grisé ${ }^{27}$, à lire sur l'eau stagnante des canaux ou les parois lépreuses des palais, ce que l'auteur lui-même appelle un "roman vaporeux de la mort» ${ }^{28}$. Venise est synonyme de mort, mais n'est que le repoussoir d'un moi qui se veut synonyme de vie. $* * *$

Heureusement, ce Barrès ivre de son moi parvient, par moments du moins, à deviner Venise au-delà du miroir : il se met à l'écoute de la ville

23. La Mort de Venise, p. 35.

24. Ibid., p. 34.

25. Ibid., p. 36.

26. Ibid., p. 103.

27. Cf. aussi, lorsqu'il contemple la mer, cette formule: "Toute cette tristesse se développait et me portait sans bruit sur des espaces immenses auxquels je servais de conscience». Venise est la conscience de Barrès, rien d'autre.

28. Ibid., p. 114. 
concrète, et cesse, ici ou là, de cajoler la mort, pour observer la vie ${ }^{29}$. Ses plus grandes réussites d'écriture ne vont pas sans cet humour qui est une des formes, et non la moindre, de la distance à soi (qui lui manque souvent cruellement). Ainsi cette belle métaphore qui joue d'une double manière sur les pigeons de la place Saint-Marc:

Il y a, tout au bas, dans Venise, une population débonnaire, naïve, ignorante du mal: de vrais pigeons. Oui, des pigeons. Le mouvement de l'oiseau, son frisson qui monte jusqu'à son cou en soulevant un peu son duvet, c'est le geste de la Vénitienne écartant soudain les coudes pour rouler son châle sur la nuque, pour mieux en disposer les plis.

Ou cette autre phrase, à l'ironie profonde, si je puis dire, et l'une des plus précieuses de l'œuvre à mes yeux:

Nous rentrâmes dans la ville avec un sentiment de stupeur et de regret, avec la courbature générale que dut avoir Lazare à sa résurrection ${ }^{30}$.

De même, il arrive qu'on perçoive, dans cette œuvre, l'expression d'une souffrance, qu'on entrevoie le surgissement d'une faille. Ainsi, dans ce passage qui confesse que «nul homme ne s'est jamais guéri [...] Celui que trahirent une fois des amis n'est plus un beau fruit sans meurtrissure, celui qui subit un échec, une offense ne partira plus jamais comme un beau trait, spontanément à l'appel qui l'émeut. Je le vois qui tâtonne, hésite. Le son n'a plus sa pureté exquise " ${ }^{31}$. On peut penser que Barrès parle de sa propre personne, et que Venise fait remonter en lui une conscience authentique du paradis perdu. De même a-t-il le sentiment aigu de la fragilité de la beauté, comme dans ce passage:

Pendant longtemps notre plaisir, devant ce chef-d'œuvre du gothique vénitien [il s’agit de la Cà d'Oro], eut la qualité douloureuse qu'inspire une beauté imprudente, si elle n'oppose aux fièvres que ses grâces ${ }^{32}$.

29. Cf., chez Barrès, la description des sons de Venise (p. 25 sq).

3o. Ibid., p. 54.

31. Ibid., p. 111 sq.

32. Ibid., p. 22. 
Ailleurs, on lit cette exclamation: "Frappe donc, ô beauté!» ${ }^{33}$. Expression distante et ironique dans son emphase même, mais dont le sens, à tout prendre, est plutôt grave.

Lassurance de soi, chez le voyageur Barrès, n'est donc pas toujours entière, sa capacité de voir le monde est bien réelle, et ses failles, bien sûr, nous le rendent plus attachant que ses certitudes souvent trop arrogantes. Mais si le doute, la mélancolie, voire la douleur menacent parfois le visiteur de Venise-la-morte, si l'humour et le sens de l'observation lui permettent de s'élever au-dessus du narcissisme, il reste qu'il ne s'en échappe pas décidément; et que pour l'essentiel, ses failles ne deviennent pas déchirure; ses tensions ne deviennent pas écartèlement. Au bout du compte, nulle discordance essentielle ne le sépare de l'univers, puisque l'univers, c'est lui-même. Et la "mort de Venise» n'est rien d'autre que la «vie de Barrès». Vision solidement, résolument, absolument moniste. $* * *$

Barrès parle beaucoup des fièvres de Venise, et du paludisme. Chez Thomas Mann et sa Mort à Venise, qui date de 1912, neuf ans après La Mort de Venise, ce sera le choléra. La trame de ce récit célèbre est si connue que j'aurais honte de la rappeler en plus de quelques mots: le fameux écrivain Gustav Aschenbach, réputé pour l'admirable maîtrise de son art, mais aussi de sa vie - et de la vie - éprouve brusquement le besoin de quitter l'Allemagne. Il s'installe à l'Hôtel des Bains, sur le Lido, et là, à sa propre angoisse, il tombe en stupeur, puis tombe en amour devant un très jeune garçon polonais d'une extrême beauté, prénommé Tadzio. De plus en plus fou de lui, il le poursuivra partout, à distance, sans jamais l'aborder vraiment. Le choléra survient. Les autorités vénitiennes dissimulent la vérité, afin d'éviter la fuite des touristes. Aschenbach, lors d'une de ses traques dans la ville aux odeurs méphitiques, mangera des fruits avariés, et mourra sur la plage, à la fois du choléra et de la beauté de Tadzio, qu'il n'a jamais atteinte. Et comme le dit ironiquement la dernière phrase du récit, la nouvelle de sa mort est accueillie, partout dans le monde, "avec une religieuse émotion". Sous-entendu: personne ne saura jamais de quoi est réellement mort cet homme si digne et si respectable, cet artiste si maîtrisé.

Il n'est pas anecdotique de souligner l'ancrage de cette nouvelle dans la réalité historique, l'histoire collective et l'histoire personnelle de Thomas

33. Ibid., p. 18 sq. 
Mann. J'ai déjà indiqué que cet ancrage était très solide, plus solide que les pieux de Venise dans le limon de la lagune. Le choléra, tout d'abord, est un fait historique. Il ravagea toute l'Europe en 1911, et n'épargna pas Venise. Mais surtout, Katia Mann, la femme de Thomas Mann, indique dans ses souvenirs qu'absolument tous les épisodes de ce récit furent vécus, en particulier les rencontres avec divers personnages inquiétants, qui sont autant d'annonciateurs de la mort: un horrible vieux beau, un gondolier muet et un chanteur des rues répugnant et cynique.

Katia Mann confirme aussi l'admiration que son digne époux portait à un tout jeune garçon présent dans leur hôtel. Bien entendu, elle met au compte de l'invention romanesque la transformation de cette admiration toute platonique en une passion féroce. Quant à l'issue fatale de cette passion, il se trouve que la famille Mann, prudente, quitta Venise en même temps que la famille polonaise dont le plus jeune représentant fascinait l'écrivain ${ }^{34}$. Tout cela pour dire que le statut du récit intitulé $\mathrm{La}$ Mort à Venise n'est pas si différent du statut de La Mort de Venise de Barrès. Barrès recourt à un "je» littéraire, qui est lui sans être tout à fait lui. Thomas Mann recourt à un «il» littéraire qui n'est pas lui mais qui l'est tout de même passablement, jusqu'à la mort non comprise.

Mais venons-en à ce qui fait à mes yeux la différence la plus radicale entre les deux œuvres. Autant la vision de Barrès est moniste, et consiste à prendre Venise pour miroir d'un moi qui se parfait devant elle, autant la vision de Thomas Mann est dualiste. Venise, chez lui, est le lieu d'une révélation qui déchire le moi, le divise, l'affole et finalement le détruit. Ici, le fait que nous soyons en présence d'un roman, dans lequel la conscience de l'auteur surplombe celle de son héros, n'est pas sans importance. Néanmoins, la conscience n'est pas le fait de l'auteur seul. Le héros lui-même fait pleinement l'expérience du dualisme qui est au principe de la pensée de Thomas Mann. Je pourrais presque dire, anticipant sur la suite de mon propos, qu'Aschenbach, avant d'arriver à Venise, cultivait une vision moniste du monde, et que la dualité du réel et des êtres humains va le frapper de plein fouet. Venise va lui donner, de lui-

34. Des recherches plus poussées ont d'ailleurs permis de donner un nom à ce jeune garçon. Il s'agirait du baron Wladyslaw Moes (1900-1986), que sa mère ou ses sœurs appelaient souvent Adzio ou Wadzio, et qui s'est lui-même reconnu, plus tard, dans le Tadzio de Thomas Mann. 
même, une image véridique, mais d'une vérité déchirée, insupportable, et qu'il avait toujours refusée.

Le monde est duel, l'homme est duel. Apollon s'y heurte à Dionysos, l'univers chrétien à l'univers païen, le jour à la nuit, la raison à la folie, la discipline à la licence, la forme pure à l'abîme sans fond. Le drame d'Aschenbach est, si je simplifie un peu grossièrement, de s'être pris pour Goethe, ou plutôt de s'être mépris sur Goethe. Car Goethe lui-même a connu la tentation dionysiaque, les affres des amours impossibles et la fascination des gouffres ${ }^{35}$. Le Faust témoigne amplement que cet olympien présumé connaissait les puissances de la nuit et de la mort ${ }^{36}$. L'erreur et le drame d'Aschenbach, c'est donc d'avoir cru et donné à croire qu'Apollon peut régner sans partage sur Dionysos, que l'art peut se déployer dans une sérénité suprême en oubliant à jamais les abîmes qui l'ont fait naittre; enfin, que la lumière peut être belle dans l'ignorance de la nuit. La rencontre de Tadzio, la découverte d'une beauté fulgurante mais interdite, la fascination d'un amour sans issue heureuse, et même sans nulle issue, tout cela, Aschenbach le vit comme une épouvantable et délicieuse régression, une visite aux abîmes, transgressive et palpitante, lui qui précisément avait, sa vie durant, "dénoncé toute sympathie avec les abîmes ${ }^{37}$ ». Mais de cette visite, il ne pourra remonter vivant, parce qu'il n'y était pas préparé. Celui qui a méconnu ses propres gouffres ne peut y descendre sans mourir.

Venise, dans l'œuvre de Thomas Mann, est aussi mortifère que chez Barrès; elle l'est même davantage, parce que plus concrètement: le choléra tue vraiment, et Aschenbach mourra, à la fois de cette maladie et de sa maladie d'amour. Mais on peut décidément dire que Venise, qui pour Barrès est en quelque sorte le lieu d'une vérification et d'une exaltation de son moi unitaire, sera pour Aschenbach le lieu d'une révélation ravageuse, d'une mise en question de tout son être, d'un choc mortel; peut-être pas l'apparition d'une vérité absolument nouvelle, mais pour

35. D'ailleurs, il est singulier de noter (je ne sais si c'est une coïncidence ou non) que Goethe, lorsqu'il s'est embarqué pour son fameux voyage en Italie, s'est littéralement enfui de Weimar, à l'insu de presque tous, poussé par un démon intérieur. Même lui!

36. On peut d'ailleurs ajouter que, selon Thomas Mann lui-même, La Mort à Venise aurait dû être d'abord le récit de l'aventure du vieux Goethe amoureux, à Marienbad, de la toute jeune Ulrike von Lewetzov, amour évidemment sans espoir, et qui fut (donc) à l'origine de l'extraordinaire Élégie de Marienbad.

37. Cf. Th. Mann, La Mort à Venise, p. 125. 
le moins la résurgence d'une vérité qu'il n'aurait jamais dû méconnaître: nous sommes duels, et de la manière la plus douloureuse qui soit.

Nous sommes duels, redisons-le, en ce sens fondamental que nous sommes attirés à la fois par la nuit et la lumière, par Apollon et par Dionysos; nous sommes à la fois pétris de morale chrétienne et de rêves païens, fervents d'idéal ascétique et travaillés de délires érotiques. Et soit dit en passant, de longs passages de la nouvelle de Thomas Mann invoquent et même citent le Phèdre de Platon, puisque l'«amour grec», ainsi qu'il est convenu de l'appeler dans un tel contexte, est justifié par le Socrate de Platon, et même glorifié: il est indissociable d'une ontologie de l'Éros, à laquelle s'opposera l'ontologie de l'Agapè chrétienne, et ce qu'il est également convenu d'appeler la morale chrétienne. Cette morale interdit à Aschenbach de toucher à Tadzio, alors que la morale du Phèdre, au contraire, lui enjoindrait d'en faire son «éromène». La dualité paganisme-christianisme, Éros-Agapè, qui dépasse et englobe en somme celle de Dionysos et Apollon, est donc vécue par Aschenbach dans toute sa violence.

Mais la pire des dualités que découvre le héros de Thomas Mann est peut-être encore ailleurs: nous sommes duels en ce sens terrible et radical où l'accès aux réalités les plus hautes, les plus idéales, les plus belles, passe par le gouffre, l'informe, le chaos, et se paie d'une souffrance constante, irréductible, insupportable. Souffrance que l'on subit et parfois qu'on inflige. En ce sens, d'ailleurs, La Mort à Venise, avant le Docteur Faustus, est une nouvelle version du mythe de Faust, s'il est vrai que Faust est celui qui accède aux plus hauts mystères et à la plus haute puissance, mais qu'il paie cet accès de la perte de son âme, c'est-à-dire de la complicité avec la nuit, le crime, la mort - le diable, pour tout dire. Le diable, à cause de qui Faust fait du mal et souffre le mal.

$* * *$

Et maintenant, où veux-je en venir? Où puis-je en venir? Il n'est évidemment pas question de vous proposer un concours entre l'œuvre vénitienne de Barrès et celle de Thomas Mann, ni de leur distribuer des bons et des mauvais points.

En tout état de cause, ce n'est pas la qualité littéraire de ces deux écrivains qu'il s'agit de discuter, c'est leur univers qu'il s'agit de comparer, dans la mesure où cette entreprise est réalisable. Mais après tout, c'est l'entreprise que tout lecteur fait toujours, lorsqu'il passe d'un livre à l'autre et d'un auteur à l'autre. Et ce qui lui reste d'une œuvre lue, c'est à 
la fois des images précises, et, au-delà d'elles, une tonalité fondamentale, une «teneur». Disons qu'en risquant une comparaison Barrès-Mann, je ne fais que reproduire un geste intérieur qui est celui de tout lecteur. On compare les livres comme on compare les êtres. Sans le vouloir, et nécessairement.

Je puis en outre me réclamer du grand exemple de Charles Du Bos, qui s'est tout bonnement posé, devant Thomas Mann et devant son auditoire parisien, la seule question qui compte: quelle est la relation de l'auteur allemand et de l'auteur français aux "puissances souterraines"? Comme son hôte Thomas Mann, Du Bos se souvenait évidemment, en 1926, d'une guerre abominable et toute proche encore. Or voilà qu'un grand écrivain allemand faisait le voyage de Paris. Comment ne pas songer au déchaînement qui avait été, durant quatre années, celui des "puissances souterraines» et des forces de mort? Du Bos, donc, affirmait que Thomas Mann avait dépouillé le nationalisme et les tentations mortifères. Et que Barrès, de son côté, commençait de le faire quand il est décédé. Qu'il l'aurait fait à coup sûr, s'il eût vécu.

Que faut-il en penser? Même si je ne saurais décider ce que serait devenu un Barrès vivant après 1923, je crains qu'en tout cas La Mort de Venise ne contienne pas, au contraire de La Mort à Venise, les ferments d'un changement essentiel sur ce terrain qu'on pourrait appeler métaphysique. Pourquoi? Parce qu'il faut, me semble-t-il, qu'un homme, un écrivain soit habité par la conscience d'une déchirure profonde, d'une dualité radicale entre le jour et la nuit, les forces de vie et les forces de mort, pour espérer faire obstacle, le moment venu, à ces dernières.

La Mort à Venise de Thomas Mann nous raconte en somme l'histoire d'un homme qui n'avait pas conscience de cette dualité. D'où sa chute dans le malheur et la mort. Aschenbach avait cru dénoncer «toute sympathie avec les abîmes". Il se complaisait dans une vision moniste, où tout n'était que lumière faussement goethéenne, tout n'était que maîtrise et beauté sans douleur. Le choc de la rencontre de Tadzio lui fait redécouvrir l'existence de la nuit et de l'abîme, et comprendre, à ses dépens, que toute vraie lumière suppose la nuit, exige la nuit. Thomas Mann lui-même, derrière Aschenbach, est l'homme qui a pris conscience, mais une conscience progressive, de la dualité du monde, et de sa propre dualité intérieure. C'est à ce prix qu'il pourra faire leur part à la nuit comme à la lumière, à Apollon comme à Dionysos, et assigner leur place aux "puissances souterraines», une place qui, sans les nier, les tient en 
respect, parce que l'homme est capable de les saluer sans leur complaire; de les reconnaitre sans les adorer.

J'ai envie de dire, en simplifiant sans doute à outrance, que Barrès, en tout cas le Barrès de 1903 et de La Mort de Venise, se complaît dans le culte de la nuit et des puissances souterraines, dans la contemplation d'une mort faite à sa mesure, à son bon plaisir, et qui n'est que le dépôt de son moi sur Venise; nullement l'altérité radicale, le tout autre et l'ennemi. Du coup, il ne songe pas à lui opposer cette exigence de lumière dont l'une des formes les plus nobles et les plus hautes est l'exigence de raison. Apollon est le dieu de la lumière, donc des Lumières. Or, nombreux sont les passages de La Mort de Venise (et pas seulement de cet ouvrage) qui font le constat satisfait (ou le prétendu constat) que nous sommes menés par notre inconscient, et que la raison n'est rien. Les "vérités positives", dit paradoxalement l'auteur dans sa préface, sont "dans notre profond sous-conscient ${ }^{38}$. Ou encore cette formule étrangement auto-réfutatrice: "Nous ne sommes pas les maîtres des pensées qui naissent en nous" ${ }^{39}$.

Ajoutez à ce goût de l'inconscient, à ce désir que l'inconscient nous mène, cette consécration de toute sa conscience au culte d'un moi qui met le monde à son service, pour ne pas dire qu'il se l'asservit; ajoutez ce monisme ontologique obstiné, et vous ne trouverez pas, chez ce Barrès-là du moins, de lieu pour penser les puissances souterraines, donc les tenir en respect. Vous ne trouverez pas la conscience douloureuse de ce dualisme humain, conscience qui seule nous permettra de faire, si j'ose dire, la part du diable. Dès lors, au moment où les puissances diaboliques et souterraines se manifesteront dans la guerre, pourquoi ne pas les saluer avec enthousiasme, pourquoi ne pas y voir, dans la plus tragique des méprises, des puissances de vie?

Il se peut que ma vision de Barrès, moins optimiste que celle de Du Bos, soit liée aux conditions dans lesquelles j’ai sinon découvert, du moins quelque peu approfondi cet auteur: une lecture des Scènes et doctrines du nationalisme, qui, parues en 1902, sont presque contemporaines de La Mort de Venise, et que j'ai approchées au moment où

38. Ibid., p. VII.

39. Ibid., p. 276. Cette dernière citation est à vrai dire tirée du « 2 novembre en Lorraine"; mais ce texte fait partie d'Amori et dolori sacrum. 
j'écrivais mon ouvrage sur l'affaire Dreyfus et les écrivains. On pourrait d'ailleurs trouver, dans ces Scènes et doctrines, des phrases qui consonent exactement avec certaines formules de La Mort de Venise. Ainsi : "Nous ne sommes pas maîtres des pensées qui naissent en nous " ${ }^{40}$.

Or je voudrais, pour terminer, vous citer une autre phrase de Barrès, infiniment révélatrice, à mes yeux, de tout ce que je souhaitais vous dire ici. Nous avions constaté que La Mort de Venise est habitée, d'un bout à l'autre, par des images de décrépitude, de pourrissement et de décomposition; mais nous avions constaté de même que Barrès apportait avec lui, et déposait sur la ville réelle, cette vision de décrépitude et de maladie mortelle. Au point de faire dire, à l'ensemble des poètes et musiciens qu'il institue ses garants, ce qu'ils n'ont pas vraiment dit. Bref, cette vision de la mort, cette obsession n'est que celle de l'auteur, et n'a pas grand-chose à voir avec la réalité sociale et concrète de la Venise qu'il a visitée. En un mot comme en cent, la Venise de Barrès, je le redis une dernière fois, c'est Barrès lui-même.

Si je me répète ainsi, c'est pour mieux préparer la citation que voici: «...[il] n'offrait de remarquable que les marbrures de la décomposition». Les marbrures de la décomposition! Ne croirait-on pas lire une formule tout droit sortie, elle aussi, de La Mort de Venise, décrivant peut-être un palais décrépit, voire le corps d'une prostituée vénitienne, rongé par la maladie? Eh bien non, pas exactement. Je lis maintenant la citation sous une forme plus complète:

Nous pûmes voir les scrupules, les tortillements et les nuances de cette pensée malsaine où passent les couleurs changeantes d'un poisson qui se meut. Soldat sorti de son élément, il prétendait aux plus subtiles analyses et n'offrait de remarquable que les marbrures de la décomposition ${ }^{41}$.

Cette phrase résume les impressions de Barrès au procès de Rennes, le second procès Dreyfus, en 1899, devant le témoignage d'un certain colonel Picquart, celui-là même dont le jeune Proust fera dans Jean Santeuil le héros de l'esprit libre, de la pensée juste et courageuse...

Je ne crois pas aller trop loin en affirmant que le Barrès du procès de Rennes inventait un Picquart à son goût (à son dégoût, plutôt), c'est-à-

40. Cf. M. Barrès, Scènes et doctrines du nationalisme, p. 18.

4I. Ibid., p. 135. 
dire une intelligence décomposée et pourrissante, comme il inventera, presque au même moment, une Venise non moins décomposée et pourrissante. Et je ne crois pas aventuré d'ajouter que le monisme intraitable et inexpugnable de cet écrivain lui joue décidément des tours, et l'empêche de voir dans le monde autre chose que le miroir de son moi omniprésent, totalisant, phagocytant. Il n'y a pas place en lui pour le choc de l'altérité. L'autre est toujours un faire-valoir, toujours un repoussoir, grâce à qui le moi peut se voir en majesté. Le monde? C'est la mort qui permet à Barrès, visiteur de Venise, de se sentir mieux vivre; c'est l'ennemi mortel, et peint aux couleurs d'un cadavre, qui permet à Barrès, spectateur du procès de Rennes, de se sentir conforté dans sa haine antidreyfusarde. Le phénomène est à chaque fois le même.

Qu'on me permette de préférer, à ce Barrès-là, celui qui, de loin en loin, admet que son moi n'est pas une monade inexpugnable, comme dans ces pages de La Mort de Venise où sans crier gare il nous avoue, trop brièvement, faiblesse ou désarroi; comme dans cette page soudain précieuse de son pénible compte-rendu du procès Dreyfus, où il parvient tout à coup à écrire: «Pour moi, que mes amis m'excusent, je considérais l'homme, la figure lointaine, le fantôme qui met la France en crise, et je sentais que ce nom exécré de Dreyfus représentait tout de même de la chair vivante et broyée $[\ldots]{ }^{42}$. Et voilà que Barrès, juste après cette phrase, profère le mot de "pitié», rejoignant ainsi, rejoignant enfin, dirais-je, cette «sym-pathie» que Du Bos saluait chez Thomas Mann; donnant donc raison, enfin, aux espoirs de ce critique si généreux.

Pardonnez-moi de l'avoir été moins que lui. Mais je crois trop, je vous l'ai dit, à cette vérité première de la littérature et de tous les arts: l'esthétique ne va jamais seule. Et chez un écrivain, la beauté de la langue, toujours, donne forme et force à sa vision du monde. Honneur à la perfection du style, puisqu'elle seule met en pleine lumière la vérité du monde.

Étienne BARILIER

Écrivain

Professeur émérite à l'Université de Lausanne

42. Ibid., p. 103. 


\section{BIBLIOGRAPHIE}

\section{Textes}

BArrès, Maurice, Amori et dolori sacrum, Paris, Félix Juven, 1903.

—, Scènes et doctrines du nationalisme, Paris, Editions du Trident, 1987.

-, Un homme libre, in Romans et voyages, coll. Bouquins, Laffont, 1994.

Byron, George Gordon Lord, Le Pèlerinage de Childe Harold, in Euvres complètes de Lord Byron, traduites par Benjamin Laroche, première série, Paris, Victor Lecou, 1847.

Du Bos, Charles, «Hommage à Thomas Mann», in Id., Approximations, Paris, Éditions des Syrtes, 2000 (1933), p. 661-677.

Goethe, Johann, Wolfgang, Voyage en Italie, I, Paris, Aubier, 1961.

Mann, Thomas, La Mort à Venise, trad. de Félix Bertaux et Jacques Sifwalt, Paris, Fayard (Le Livre de Poche), 1971 (1925).

—, La victoire finale de la démocratie, Gallimard, 1939.

Ruskin, John, La Bible d'Amiens, trad. et préface de Marcel Proust, Paris, Mercure de France, 1905.

—, Les Pierres de Venise, trad. de Mathilde P. Crémieux, Paris, Renouard, 1921.

\section{Travaux}

Cheval, René, Romain Rolland, l'Allemagne et la guerre, Paris, Presses Universitaires de France, 1963.

Godo, Emmanuel, La légende de Venise; Maurice Barrès et la tentation de l'écriture, Lille, Presses Universitaires du Septentrion, 1996.

Petriconi, Hellmuth, Das Reich des Untergangs, Hamburg, Hoffman und Campe, 1958. 
